\title{
Ultrasound screening in gynecological examinations: can we overcome the criticism that it is insufficient and harmful?
}

\author{
Katsuhiko Naruse ${ }^{1}$
}

Received: 15 November 2021 / Accepted: 19 November 2021

(c) The Author(s), under exclusive licence to The Japan Society of Ultrasonics in Medicine 2021

Ultrasound is performed simultaneously with vaginal cytology in many facilities during examinations focused on gynecologic tumors. Traditionally, we have relied on the pelvic examination (bimanual palpation), but its accuracy has not been high, and the influence of certain factors, such as the patient's body mass index, have been unavoidable [1]. In addition, widespread use of transvaginal ultrasonography has improved the detection of ovarian tumors and uterine fibroids.

On the other hand, it has been observed that routine ultrasonography for the detection of ovarian and uterine endometrial cancers is not entirely relevant, does not contribute to mortality reduction, is not valid, and may lead to overdiagnosis [2]. We should be aware that there is no consensus on the use of ultrasound in gynecological examinations. This is probably because of the limited ability of ultrasound diagnosis to determine whether ovarian tumors are benign or malignant and because a pathological diagnosis cannot be confirmed without operative histology. In addition, the goal of a screening test should be early detection to reduce the risk of disease and improve prognosis. However, thus far, ultrasound screening of ovarian cancer has not been able to improve outcomes, which may be due to its refractoriness [2]. On the other hand, endometrial cancer progresses slowly, and the prognosis is not so poor even when detected later based on other subjective symptoms, which is thought to reduce the priority of using ultrasonography for screening of asymptomatic individuals.

However, in Japan, where ultrasound equipment is widely available (more than in any other country worldwide), it is typical for gynecologists, most of whom are also ultrasound specialists, to expect ultrasound to be used as a screening tool in gynecological examinations in the future. We

Katsuhiko Naruse

naruse@ naramed-u.ac.jp

1 Department of Obstetrics and Gynecology, Nara Medical University, Kashihara, Japan consider that one way to overcome this challenge is by providing physicians with high-quality education, which can help achieve the best results in clinical practice while limiting costs.

The prevalence of endometrial cancer has been increasing [3]. Therefore, we will be expected to detect this disease in the future during routine medical examinations by patients or by health insurance payers. However, women sometimes avoid reporting genital bleeding due to embarrassment, and just interviewing them is not a reliable method. In addition, performing endometrial cytology on all patients is inefficient as a screening tool and causes pain. Hence, suspicious ultrasonographic findings would be useful for early detection and diagnosis of uterine endometrial cancer. Furthermore, ultrasonography has been reported to be helpful for the classification of its advanced stages [4]. On the other hand, whether early detection can contribute to patient prognosis depends on the histological type and patient's age [3]; therefore, further accumulation of data to narrow down candidates for screening is necessary.

It is important to improve the accuracy of diagnosing benign and malignant tumors using color Doppler ultrasound and other methods, particularly for ovarian tumors. The author's institution has attempted to successfully differentiate between ovarian cancer and benign endometrial (chocolate) cysts based on the shape of the finding on MRI images [5]. In addition, the treatment prognosis may improve further with advances in development of anticancer drugs, especially molecular targeted therapies. We hope that early detection of ovarian cancer will soon be good news for patients, not a death sentence.

Of course, more research and investigations need to be conducted before we can establish the validity of using ultrasonography as a screening tool. Strict attention is required to avoid overdiagnosis. The medical costs involved (including physician labor costs) cannot be ignored. As the prevalence of ovarian and uterine endometrial cancers varies with age, it is beneficial to perform thorough clinical examinations, 
or narrow down the age range or high-risk group of women who should undergo these health examinations. It is a challenging task to ensure that all patients who should come for gynecological examinations are properly examined. The use of transvaginal ultrasound probes is widespread; hence, the accuracy of diagnosis should not vary between facilities. However, the diagnostic quality is expected to become more uniform in the near future with wider application of artificial intelligence (AI) analysis. Ultrasound specialists must take the lead in overcoming these barriers so that ultrasound screening in gynecological examinations can be legitimately evaluated.

Acknowledgements The author would like to thank Professor Jun Murotsuki of Miyagi Children's Hospital for his original ideas and advice in writing this manuscript.

\section{References}

1. Padilla LA, Radosevich DM, Milad MP. Accuracy of the pelvic examination in detecting adnexal masses. Obstet Gynecol. 2000;96:593-8.
2. Henderson JT, Webber EM, Sawaya GF. Screening for ovarian cancer: updated evidence report and systematic review for the US Preventive Services Task Force. JAMA. 2018;319:595-606.

3. Shigeta S, Nagase S, Mikami M, et al. Assessing the effect of guideline introduction on clinical practice and outcome in patients with endometrial cancer in Japan: a project of the Japan Society of Gynecologic Oncology (JSGO) guideline evaluation committee. J Gynecol Oncol. 2017;28:e76.

4. Dueholm M, Hjorth IM, Dahl K, et al. Preoperative prediction of high-risk endometrial cancer by expert and non-expert transvaginal ultrasonography, magnetic resonance imaging, and endometrial histology. Eur J Obstet Gynecol Reprod Biol. 2021;263:181-91.

5. Tanase Y, Kawaguchi R, Takahama J, et al. Factors that differentiate between endometriosis-associated ovarian cancer and benign ovarian endometriosis with mural nodules. Magn Reson Med Sci. 2018;17:231-7.

Publisher's Note Springer Nature remains neutral with regard to jurisdictional claims in published maps and institutional affiliations. 